\title{
Grafted Neural Progenitor Cells Persist in the Injured Site and Differentiate Neuronally in a Rodent Model of Cardiac Arrest-Induced Global Brain Ischemia
}

\author{
Patricia Meyer, ${ }^{1-4}$ Denis Grandgirard, ${ }^{1,4}$ Marika Lehner,,4 Matthias Haenggi, ${ }^{3, *}$ and Stephen L. Leib ${ }^{1,4,{ }^{*}}$
}

Hypoxic-ischemic brain injury is the leading cause of disability and death after successful resuscitation from cardiac arrest, and, to date, no specific treatment option is available to prevent subsequent neurofunctional impairments. The hippocampal cornu ammonis segment 1 (CA1) is one of the brain areas most affected by hypoxia, and its degeneration is correlated with memory deficits in patients and corresponding animal models. The aim of this work was to evaluate the feasibility of neural progenitor cell (NPC) transplantation into the hippocampus in a refined rodent cardiac arrest model. Adult rats were subjected to $12 \mathrm{~min}$ of potassium-induced cardiac arrest and followed up to 6 weeks. Histological analysis showed extensive neuronal cell death specifically in the hippocampal CA1 segment, without any spontaneous regeneration. Neurofunctional assessment revealed transient memory deficits in ischemic animals compared to controls, detectable after 4 weeks, but not after 6 weeks. Using stereotactic surgery, embryonic NPCs were transplanted in a subset of animals 1 week after cardiac arrest and their survival, migration, and differentiation were assessed histologically. Transplanted cells showed a higher persistence in the CA1 segment of animals after ischemia. Glia in the damaged CA1 segment expressed the chemotactic factor stromal cell-derived factor 1 (SDF-1), while transplanted NPCs expressed its receptor CXC chemokine receptor 4 (CXCR4), suggesting that the SDF-1/CXCR4 pathway, known to be involved in the migration of neural stem cells toward injured brain regions, directs the observed retention of cells in the damaged area. Using immunostaining, we could demonstrate that transplanted cells differentiated into mature neurons. In conclusion, our data document the survival, persistence in the injured area, and neuronal differentiation of transplanted NPCs, and thus their potential to support brain regeneration after hypoxic-ischemic injury. This may represent an option worth further investigation to improve the outcome of patients after cardiac arrest.

Keywords: ischemic brain injury, hippocampus, neuronal progenitor cells, therapy

\section{Introduction}

$\mathrm{T}$ HE GLOBAL INCIDENCE of out-of-hospital cardiac arrest is estimated to be 55 cases/100,000 persons/year, the average survival being 7\% [1]. Among patients who are resuscitated, the leading cause of morbidity and mortality is hypoxic-ischemic brain damage [2], with half of the survivors left with cognitive deficits, in particular, memory impairments $[3,4]$. Certain brain structures are especially vulnerable to ischemic injury, such as the pyramidal neurons in the cornu ammonis segment 1 (CA1) in the hippocampus [5-7]. Loss of these cells has been associated with memory deficits, in rodents [8] as well as in humans [9]. Currently, the only treatment recommendation after cardiac arrest that improves neurological outcome is targeted temperature management [10-13]. However, this practice will never completely prevent brain damage.

During the last decades a plethora of compounds, targeting multiple stages of the hypoxic-ischemic injury cascade, have been tested as potential neuroprotective agents for brain damage following cardiac arrest. Although promising results were obtained in experimental studies, their benefits for patients with cardiac arrest could not be confirmed in clinical trials [14-16]. Thus, there is a strong need for novel therapeutic approaches.

Cell-based therapies have gained increasing attention in the past few years as a possible strategy to mitigate brain damage. Numerous experimental studies and several clinical

\footnotetext{
${ }^{1}$ Neuroinfection Laboratory, Institute for Infectious Diseases, University of Bern, Bern, Switzerland.

${ }^{2}$ Graduate School for Cellular and Biomedical Sciences, University of Bern, Bern, Switzerland.

${ }^{3}$ Department of Intensive Care Medicine, Inselspital, Bern University Hospital, University of Bern, Bern, Switzerland.

${ }^{4}$ Cluster for Regenerative Neuroscience, DBMR, University of Bern, Bern, Switzerland.

*These authors contributed equally to this work.
} 
trials were conducted to evaluate the feasibility and safety of cell transplantation in stroke [17-19], but only few for the treatment of cardiac arrest [20-25]. Their results led to a cautious optimism that this therapeutic approach could reduce brain damage and improve neurological outcome [26].

In this study, we aimed to evaluate the feasibility of neural progenitor cell (NPC) transplantation after cardiac arrest. For this, we refined our rodent cardiac arrest model [27] by increasing the duration of no-flow time (from 10 to $12 \mathrm{~min}$ ), aiming to induce more severe cognitive deficits, and describing long-term histological and neurofunctional outcomes.

\section{Materials and Methods}

\section{Animals and experimental groups}

All animal studies were approved by the Animal Care and Experimentation Committee of the Canton of Bern, Switzerland (licences BE 62/17 and BE64/17), and followed the Swiss national guidelines for the performance of animal experiments. Nine-week-old male Wistar rats were obtained from Janvier Labs (Le Genest-Saint-Isle, France) and kept in individually ventilated cages with controlled 12-h light/12-h dark cycles at $22^{\circ} \mathrm{C} \pm 2{ }^{\circ} \mathrm{C}$. Food and water were provided ad libitum.

A total of 135 animals were included in the study. Animals underwent cardiac arrest $(n=94)$ or served as controls $(n=41)$. Histological analysis was assessed 1 week after surgery in 13 cardiac arrest and 7 control animals. Morris water maze was performed on two different subsets: 13 cardiac arrest and 10 control animals 4 weeks after surgery, and 13 cardiac arrest and 13 controls 6 weeks after surgery. After conclusion of the behavioral experiments, histological analysis was performed on all animals. The remaining 34 cardiac arrest and 11 control animals were enrolled in the second study, to evaluate the feasibility of stereotactic cell transplantation after cardiac arrest. Three animals did not survive stereotactic surgery, the remaining were histologically assessed after 1,2, and 6 weeks.

\section{Induction of cardiac arrest and resuscitation}

Procedures are described briefly, details are provided in the SupplementaryData.

Cardiac arrest induction and resuscitation were performed as described previously [27]. Briefly, after induction of anesthesia, animals were orotracheally intubated and catheters were inserted in the femoral artery for blood pressure monitoring and blood sampling, and in the jugular vein for drug administration. Cardiac arrest was induced by i.v. injection of potassium chloride $(\mathrm{KCl})$ (Kalium Chloratum Sintetica; Sintetica SA, Switzerland) and esmolol (Esmolol-Orpha; OrPha Swiss GmbH, Switzerland). After $12 \mathrm{~min}$, rats were resuscitated by adrenaline and calcium injection and manual chest compressions. Control animals (sham group) underwent the same surgical interventions, without cardiac arrest induction.

\section{Assessment of learning and memory function by Morris water maze}

One week before learning and memory assessment, an open-field test was conducted to test spontaneous locomotor activity of animals. Rats were placed in an open-field arena
$(90 \times 90 \times 40 \mathrm{~cm})$ and recorded during $5 \mathrm{~min}$ with the video tracking system Ethovision ${ }^{\circledR}$ XT-11 (Noldus Information Technology, Wageningen, the Netherlands). Velocity and total distance moved by the animals were analyzed as readout for general locomotor activity.

Learning and memory performance were assessed by Morris water maze 4 or 6 weeks after cardiac arrest as previously described [28]. Swimming patterns of rats were recorded with the video tracking system Ethovision XT-11 (Noldus Information Technology). The tank (1.8 m diameter) was filled with darkened water and the surface was virtually divided into four quadrants. An adjustable black platform $(16 \times 13 \mathrm{~cm})$ was placed in the center of the first quadrant, $0.5 \mathrm{~cm}$ below the water surface, not visible for swimming rats. Three entry zones were marked outside the pool and several distal cues were placed on three walls surrounding the water maze.

During 5 days, animals were trained with four trials per day to find the hidden platform in a fixed position. Rats were put into the water facing the tank wall at one of the entry zones, which was determined by randomization. If an animal found the platform within $90 \mathrm{~s}$, it was allowed to stay on it for $20 \mathrm{~s}$ before continuing with the next trial. If the rat did not find the platform within $90 \mathrm{~s}$, it was guided there by hand and was allowed to stay on it for $20 \mathrm{~s}$. The parameter evaluated for training trials was latency to reach platform. On day 1 , the first training trial was considered "day 0." Probe trials without the platform were conducted on the first before the start of training and on day 5 before and after training trials. For these trials, time spent in the target quadrant (where platform was positioned during training) was analyzed. All measurements for swimming path were automatically recorded and computed by the video tracking system.

\section{Isolation and culture of NPCs}

Lewis rats expressing green fluorescent protein (GFP) [29] were kindly obtained from Prof. Kobayashi (Division of Organ Replacement Research, Center for Molecular Medicine, Jichi Medical School, Japan) and bred with female wild-type Lewis rats obtained from Charles River Laboratories, Germany. For the NPC isolation from GFPexpressing rat embryos, pregnant Lewis females were sacrificed by a lethal dose of pentobarbital $(150 \mathrm{mg} / \mathrm{kg}$, i.p.) on fetal day 16. Embryonic sacs were removed from the uterus and the embryos transferred into ice-cold dissection medium consisting of HBSS (H6648; Sigma-Aldrich, Switzerland), antibiotic-antimycotic $1 \times(15240-096$; Gibco, Thermo Fisher Scientific; ), and $6 \mathrm{mg} / \mathrm{mL} 45 \%$ glucose (G8769; SigmaAldrich). GFP-expressing embryos were identified using a UV lamp, brains removed and the hippocampal tissue dissected as described in [30].

Using a sterile scalpel, the tissue was cut into small pieces and incubated in $0.05 \%$ trypsin/EDTA (L2143; Bioswisstech, Switzerland) for $10 \mathrm{~min}$ at $37^{\circ} \mathrm{C}$. After adding an equal volume of trypsin inhibitor $1 \mathrm{mg} / \mathrm{mL}$ (T6522; SigmaAldrich) and centrifugation for $5 \mathrm{~min}$ at $220 \mathrm{~g}$, the pellet was resuspended in $0.2 \mathrm{~mL}$ serum-free medium [SFM: DMEM/ F12 + L-Glutamine (11320-074; Gibco, Thermo Fisher Scientific) and $15 \mathrm{mM}$ HEPES (own production)]. By gently pipetting up and down with differently sized pipette tips, cells were then mechanically dissociated. The obtained 
single-cell suspension was cultured at a concentration of $5 \times 10^{4}$ cells/mL in poly-HEMA-coated flasks (P3932; Sigma-Aldrich) in SFM supplemented with $20 \mu \mathrm{L} / \mathrm{mL}$ B27 $50 \times(17504-044$; Thermo Fisher Scientific), $10 \mathrm{ng} / \mathrm{mL}$ basic fibroblast growth factor (100-18B; PeproTech, UK), and $10 \mathrm{ng} / \mathrm{mL}$ epidermal growth factor (AF-100-15; PeproTech).

Cells were grown to neurospheres at $37^{\circ} \mathrm{C}$ in $5 \% \mathrm{CO}_{2}$ with medium changed every 2-3 days. After 6-8 days of culture, spheres were dissociated chemically and mechanically into single cells as described above and concentrated to $5 \times 10^{7}$ cells $/ \mathrm{mL}$ in phosphate-buffered saline (PBS) for transplantation.

\section{Stereotaxic surgery}

One week after cardiac arrest and resuscitation or sham surgery, GFP-expressing NPCs were transplanted into both hippocampi. Animals were anesthetized with $0.15 \mathrm{mg} / \mathrm{kg}$ medetomidine (Domitor; Provet AG, Switzerland), $2 \mathrm{mg} / \mathrm{kg}$ midazolam (Dormicum; Roche, Switzerland), and $5 \mu \mathrm{g} / \mathrm{kg}$ fentanyl (Fentanyl Sintetica; Sintetica SA) administered s.c. After surgical anesthetic depth was reached, $20 \mu \mathrm{L}$ lidocaine $1 \%$ (Lidocain $\mathrm{HCl}$; Bichsel AG, Switzerland) was administered s.c. for local anesthesia, and the rats were fixed in a stereotaxic apparatus (Kopf Instruments, Model 902 Dual Small Animal Stereotaxic Instruments). Oxygen was supplied through a nose mask throughout surgery, and body temperature was kept at $37^{\circ} \mathrm{C}$ with a temperaturecontrolled heating mat.

After exposing the skull by a midline incision and removal of skin, muscle, and periosteum, two holes were drilled at the following coordinates starting from bregma: AP: $-4.0 \mathrm{~mm}, \mathrm{~L}: \pm 2.3 \mathrm{~mm}$, and $-2.7 \mathrm{~mm}$ [31]. The NPC suspension $\left(5 \times 10^{7}\right.$ cells $\left./ \mathrm{mL}\right)$ was injected into both hippocampi, $2 \mu \mathrm{L}$ each, using a $10 \mu \mathrm{L}$ Hamilton syringe. Drill holes were sealed with bone wax (Ethicon LLC) and the incision sewed. Approximately, $2 \mathrm{mg} / \mathrm{kg}$ Ropivacaine (ROPIvacain Fresenius; Fresenius Kabi, Switzerland) was injected s.c. for local anesthesia after surgery, and $100 \mathrm{mg} / \mathrm{kg}$ ceftriaxone (Rocephine; Roche) s.c. for postoperative prophylaxis. Anesthesia was then reversed with $0.75 \mathrm{mg} / \mathrm{kg}$ atipamezole (Revertor; Virbac AG, Switzerland), $0.2 \mathrm{mg} / \mathrm{kg}$ flumazenil (Flumazénil Labatec; Labatec Pharma AG, Switzerland), and $0.05 \mathrm{mg} / \mathrm{kg}$ buprenorphine (Temgesic; Reckitt Benckiser AG, Switzerland) administered s.c., and animals were kept on a heating mat until fully awake.

\section{Histological assessments}

For histological damage assessment, animals were sacrificed at given time points by a pentobarbital (Esconarkon; Streuli Pharma, Switzerland) overdose ( $150 \mathrm{mg} / \mathrm{kg}$, i.p.). After perfusion with $4 \%$ paraformaldehyde (PFA) in PBS, brains were removed and further fixed in $4 \%$ PFA overnight at $4^{\circ} \mathrm{C}$. They were then transferred to $18 \%$ sucrose in PBS solution and stored at $4^{\circ} \mathrm{C}$ until processed further.

Assessment of degenerating neurons. Coronal cryosections of the hippocampus $(10 \mu \mathrm{m})$ obtained by systematic sampling (every 45th section collected) were stained with Fluoro-Jade B (FJ) (AG310; Merck, Germany) to identify degenerating neurons 1 week after surgery. Briefly, cryosections were hydrated in $94 \%$ and $70 \%$ ethanol for 3 and
$1 \mathrm{~min}$, respectively, and after rinsing in deionized water, they were incubated in a $0.06 \%$ potassium permanganate solution for $15 \mathrm{~min}$. After rinsing, sections were stained with a $0.001 \%$ Fluoro-jade B (AG310; Merck) solution for $30 \mathrm{~min}$ in the dark. This solution was freshly prepared from a $0.01 \%$ stock solution in $\mathrm{dH}_{2} \mathrm{O}$ and $0.1 \%$ acetic acid. After rinsing 3 times, sections were stained with 4',6-diamidino2-phenylindole (DAPI) and dried in the dark. Before embedding in DPX (06522; Merck), they were cleared in xylene twice for $2 \mathrm{~min}$. From every brain, five sections spanning the hippocampus of both hemispheres were imaged with a Zeiss fluorescent microscope (AxioImager M4; Zeiss, Germany) and five pictures were taken from every CA1 segment with a $200 \times$ magnification. Thus, a total of 50 pictures were quantified per animal. Using Image J 1.45I software (Wayne Rasband, National Institute of Health), the CA1 segment length was determined and the FJpositive cells were quantified. Blinding of the investigator was not performed, given the obvious difference between cardiac arrest and sham groups, that is, the massive presence of degenerating cells in cardiac arrest animals only.

Assessment of surviving neurons. For quantification of surviving neurons, hippocampal cryosections sampled using an identical protocol as above were stained with neuronal nuclear antigen antibody (NeuN, FOX3 antibody, ABN51; Merck) (see below for protocol), specific for mature neurons, and the whole CA1 segment was imaged $(100 \times$ magnification) for a total of 50 pictures per animal. Quantification of neurons was performed using ImageJ software as described above. The investigator conducting the quantification was blinded to the animal groups and time points.

Assessment of transplanted cells. For histological analysis of brains after cell transplantation, every eighth coronal cryosection $(10 \mu \mathrm{m})$ was collected ( $\sim 40$ sections/animal) and stained with different antibodies. See below for antibodies and protocols. For the quantification of animals with grafted cells in the CA1 segment, every section was checked for GFP-expressing cells in this area, thereby only cells not located at the injection site or within the migration path toward the dentate gyrus were considered. This evaluation was performed blinded to the animal groups.

Immunofluorescent staining. Immunofluorescent staining was performed with a Shandon Sequenza staining rack (Thermo Fisher Scientific). Sections were permeabilized for 5 min with $0.1 \%$ Triton $X-100$ in PBS and then blocked with $0.01 \%$ Triton $\mathrm{X}-100+2 \% \mathrm{BSA}$ for $1 \mathrm{~h}$ at room temperature. Primary antibodies (Table 1) were diluted in the same solution and added $1 \mathrm{~h}$ at $37^{\circ} \mathrm{C}$ or overnight at $4^{\circ} \mathrm{C}$. Slides were then rinsed 3 times with PBS and secondary antibodies (Table 1) were added for $2 \mathrm{~h}$ at room temperature. After rinsing again 3 times with PBS, samples were mounted using Fluoroshield containing DAPI (F6057; Sigma-Aldrich).

\section{Statistical analysis}

Statistical analyses were performed with GraphPad Prism 7 (GraphPad Software, Inc.). If not stated otherwise, results are presented as mean \pm standard deviations. An unpaired Student's $t$ test was used to compare data between two groups, and a two-way ANOVA was performed to analyze repeated measures of cardiac arrest versus sham-operated animals (probe trials of Morris water maze). The association 
Table 1. Antibodies Used in the Study

\begin{tabular}{llrl}
\hline & Species & Dilution & \\
\hline Primary antibody & & Company \\
FOX3/NeuN & Rabbit & $1: 1,000$ & Merck, Germany, ABN51 \\
SOX2 & Rabbit & $1: 500$ & Abcam, UK, ab97959 \\
Iba1 & Rabbit & $1: 500$ & Wako, Japan, 019-19741 \\
Olig2 & Rabbit & $1: 1,000$ & Abcam, UK, ab109186 \\
CXCR4 & Rabbit & $1: 100$ & Abcam, UK, ab2074 \\
GFAP & Mouse & $1: 500$ & Merck, Germany, MAB360 \\
BIII-tubulin & Mouse & $1: 1,000$ & Promega, United States, G712A \\
Vimentin & Mouse & $1: 100$ & Sigma Aldrich, Switzerland, V6630 \\
SDF-1, APC-conjugated & Mouse & $1: 100$ & R \& D Systems, United States, IC350A \\
Secondary antibody & & & \\
Anti-rabbit Cy3 & Goat & $1: 1,000$ & Jackson Immuno Research, UK, Cy3 111-165-144 \\
Anti-rabbit AF647 & Goat & $1: 500$ & Thermo Fisher Scientific, United States, A-21245 \\
Anti-mouse Cy3 & Donkey & $1: 1,000$ & Jackson Immuno Research, UK, Cy3 715-165-151 \\
\hline
\end{tabular}

APC, allophycocyanin; CXCR4, CXC chemokine receptor 4; GFAP, glial fibrillary acidic protein; Iba1, ionized calcium binding adaptor molecule 1; SDF-1, stromal cell-derived factor 1; SOX2, SRY-box transcription factor 2.

between the presence of transplanted cells within the CA1 segment and whether the animals underwent cardiac arrest or not were determined using Fisher's exact test. A twosided $P<0.05$ was considered statistically significant, with $P<0.05$ (*), $P<0.01$ (**), $P<0.001$ (***), and $P<0.0001$ $(* * * *)$.

\section{Results \\ Survival after cardiac arrest}

Cardiac arrest was induced in a total of 94 animals. All, but one animal could be resuscitated. Return of spontaneous circulation (ROSC) was achieved within $60.5 \pm 30.3 \mathrm{~s}$, and an average dose of $17.1 \pm 6.2 \mu \mathrm{g} / \mathrm{kg}$ adrenaline was injected. Of the resuscitated animals, nine died spontaneously after ROSC or extubation. Ten animals had to be euthanized during the following $24 \mathrm{~h}$, due to insufficient recovery after surgery, leading to an overall survival rate of $78.7 \%$.

After surgery, all animals lost weight and while the cardiac arrest groups started to regain weight after 4-5 days, the sham-operated animals regained weight by the second day.

\section{CA1 degeneration following cardiac arrest}

FJ staining 1 week after surgery revealed a massive increase in neuronal degeneration within the CA1 pyramidal cell layer after global brain ischemia (98.7 \pm 9.4 FJ-positive cells $/ \mathrm{mm}, n=4)$ compared to sham surgery $(0.3 \pm 0.2 \mathrm{FJ}$ positive cells/mm, $n=5 ; P<0.0001$ ) (Fig. 1A). FJ-positive cells were found exclusively in the CA1 segment of the hippocampus, while none was found in the CA3 segment (Supplementary Fig. S1A). To investigate whether this cell loss was permanent, we further quantified surviving neurons 1, 4, and 6 weeks after surgery. Assessment of NeuN staining after 1 week confirmed the damage with a severely reduced number of mature neurons in the CA1 segment $(73.5 \pm 36.4$ NeuN-positive cells $/ \mathrm{mm}, n=9)$ compared to sham $(186.5 \pm 7.9$ NeuN-positive cells/mm, $n=2, P<0.01)$ (Fig. 1B, C). The CA3 segment of ischemic animals was completely unaffected (Supplementary Fig. S1B). Cell loss persisted 4 weeks after surgery with $86.1 \pm 36.4$ NeuN- positive cells $/ \mathrm{mm}$ (cardiac arrest, $n=8$ ) versus $172.4 \pm 1.0$ NeuN-positive cells/mm (sham, $n=2, P<0.05$ ), and after 6 weeks, $62.5 \pm 40.0 \mathrm{NeuN}$-positive cells $/ \mathrm{mm}$ (cardiac arrest, $n=9$ ) versus $164.0 \pm 22.5 \mathrm{NeuN}-$ positive cells/mm (sham, $n=2, P<0.01)$. Overall, the number of surviving neurons in the CA1 segment after global brain ischemia did not evolve up to 6 weeks after the insult, and persisted at a stable low level (Fig. 1B).

Microglia accumulation shown by ionized calciumbinding adapter molecule 1 immunoreactive cells 5 days after injury [27] persisted until 4 weeks after surgery in the damaged area (Fig. 1E). The amoeboid-like morphology of microglia, characteristic for an activated state [32], was well demarcated from the surrounding healthy cortical tissue with strong ramification and long processes of resting microglia [32]. Furthermore, glial fibrillary acidic protein-positive astrocytes displaying a reactive, hypertrophic phenotype [33] were abundant in the injured area (Fig. 1F), while astrocytes in unaffected sectors such as the dentate gyrus displayed a resting morphology.

\section{Transient spatial memory deficits after cardiac arrest and resuscitation}

After recovery from cardiac arrest and resuscitation, animals did not show significant locomotor impairments compared to controls, as determined in an open-field test performed in the week preceding the behavioral experiments (Supplementary Fig. S2), and confirmed by unaffected swimming velocity during Morris water maze test (Supplementary Fig. S3).

Four weeks after surgery, learning ability was observed, both in controls (sham, $n=10$ ) and ischemic animals (cardiac arrest, $n=13$ ), indicated by the decrease in time to find the platform over the 5 training days (Fig. 2A). Spatial memory performance was assessed by probe trials without platform. During the first probe trial on day 5, cardiac arrest animals spent significantly less time in the target quadrant $(24.0 \% \pm 6.5 \%$ of the total time) compared to controls $(32.2 \% \pm 9.4 \%, P<0.05)$, indicating a deficiency in memorizing the platform's location (Fig. 2B). This difference was 
A

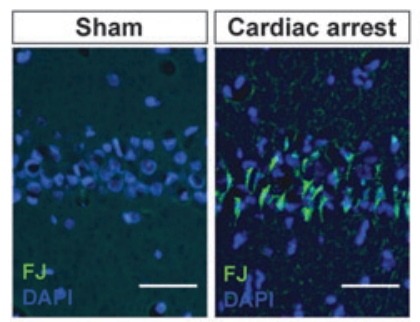

B
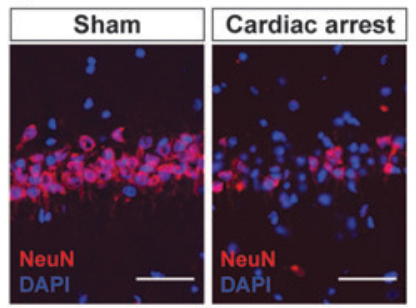
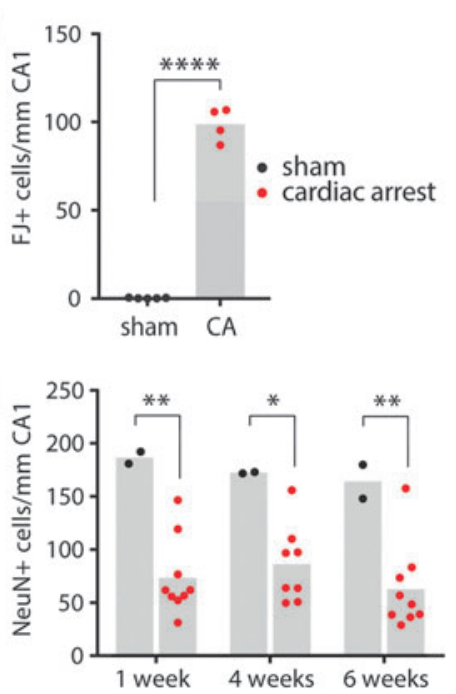

C

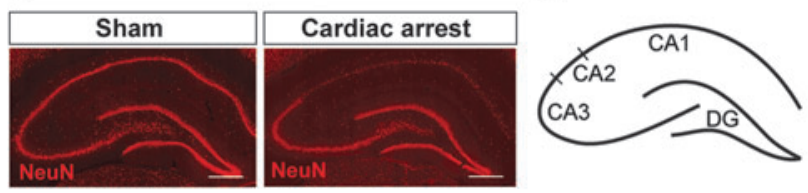

E
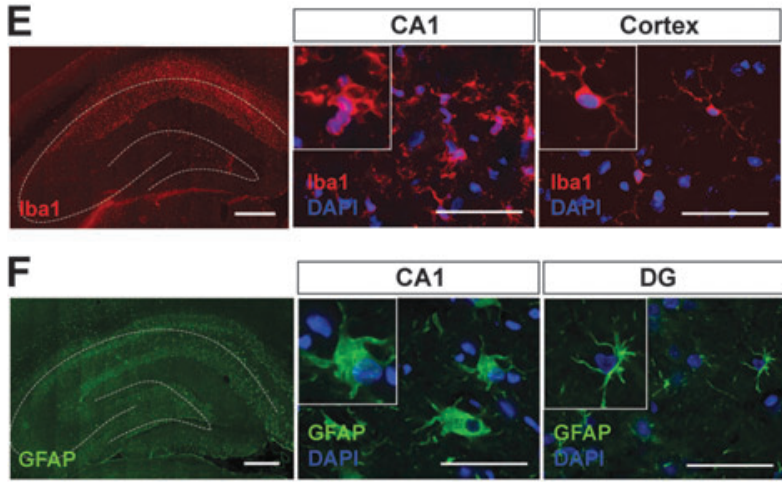

FIG. 1. Histological damage assessment after cardiac arrest and resuscitation. FJ staining 1 week after surgery, revealed the presence of degenerating neurons exclusively in the CA1 segment of cardiac arrest animals (CA) (A). Immunofluorescent staining for NeuN 1 week after resuscitation, confirmed the loss of mature neurons in the CA1 pyramidal layer after ischemia (B, C). Quantification of NeuN-expressing neurons in CA1 pyramidal layer 1, 4, and 6 weeks after surgery (B). Overview of the whole hippocampus 1 week after cardiac arrest and resuscitation or sham surgery, stained for NeuN (C). Schematic representation of the main hippocampal structures CA1-3 and DG (D). Immunofluorescent staining for glial cell markers revealed a strong inflammatory response in the damaged CA1 segment 4 weeks after the insult $(\mathbf{E}, \mathbf{F})$. Iba1-expressing microglia (E) and GFAP-positive astrocytes (F) were numerously present, and exhibited a reactive phenotype, in contrast to unaffected areas (cortex and DG). Scale bars $50 \mu \mathrm{m}$, except (C) and (E, F, whole hippocampus) $500 \mu \mathrm{m}$. CA1-3, cornu ammonis segments 1-3; DG, dentate gyrus; FJ, Fluoro-Jade B; GFAP, glial fibrillary acidic protein. ${ }^{*} P>0.05 ; * * P<0.01 ; * * * * P<0.0001$.

still present during the second probe trial performed immediately after training, without reaching significance, indicating short-term memory deficits may have developed as well. Overall, ischemic animals exhibited an impaired memory performance compared to controls (two-way ANOVA, $P<0.05)$. Six weeks after cardiac arrest and resuscitation, learning ability of the two groups was comparable (Fig. 2C), and both groups spent a similar amount of time in the target quadrant during probe trials (Fig. 2D). In summary, we documented significant memory deficits 4 weeks after global brain ischemia, which could not be detected anymore 2 weeks later.

\section{Transplanted NPCs survive up to 6 weeks and are increasingly found in CA1 segment after ischemia}

We found viable cells up to 6 weeks after transplantation (Fig. 3). After 1 week, grafted cells were found in the CA1 segment of $40 \%$ of ischemic animals $(n=2 / 5)$, whereas none was detected in this region in controls $(n=0 / 5)$. One week later, $75 \%$ of injured animals presented GFPexpressing cells in the segment of interest $(n=6 / 8)$, compared to $16 \%$ of sham animals $(n=1 / 6)$. Together, in $62 \%$ of ischemic animals, GFP-expressing cells were found in the CA1 segment compared to $9 \%$ for controls $(P<0.05)$ (Fig. 3D), indicating an increased propensity of grafted cells to persist in the CA1 segment after injury. Six weeks after transplantation, GFP-expressing cells were found in $78 \%$ of cardiac arrest animals $(n=7 / 9)$. Numerous GFPexpressing cells displayed a neuronal morphology in the CA1 segment (Fig. 3B and F and Supplementary Fig. S4B).
Especially after 6 weeks, these cells were found to align along the pyramidal cell layer in some animals (Fig. 3E and Supplementary Fig. S4C). In several instances, transplanted cells also accumulated along the corpus callosum with migration of individual cells toward the CA1 segment (Supplementary Fig. S4A, B). However, more cells still migrated toward their endogenous niche at the dentate gyrus where they accumulated along the granular cell layer in both ischemic (Fig. 3A) and control animals (Fig. 3C).

To identify an underlying molecular mechanism sustaining the observed persistence of cells in the injured CA1 segment, the stromal cell-derived factor $1 / \mathrm{CXC}$ chemokine receptor 4 pathway (SDF-1/CXCR4) was investigated, known to be pivotal for the migration of neural stem cells toward injured brain regions [34]. One week after cardiac arrest, at the time point of NPC transplantation, microglia and astrocytes accumulating in the damaged CA1 segment expressed the chemotactic molecule SDF-1 (Fig. 4A, B). Furthermore, its receptor CXCR4 was found to be expressed in cultured NPCs, at the time point of transplantation (Fig. 4C) as well as 2 weeks later in the brain parenchyma (Fig. 4D).

Six weeks after transplantation, we found a considerable number of GFP-positive cells expressing the mature neuron marker NeuN, particularly in cells located in the CA1 segment (Fig. 5A). Other grafted cells were either positive for the immature neuron marker $\beta$ III-tubulin (Fig. 5B) or for stem and progenitor cell markers such as SRY-box transcription factor 2 (SOX2) (Fig. 5D) and vimentin (Fig. 5C). Interestingly, most GFP-expressing cells found in the granular layer of the dentate gyrus co-expressed SOX2, 

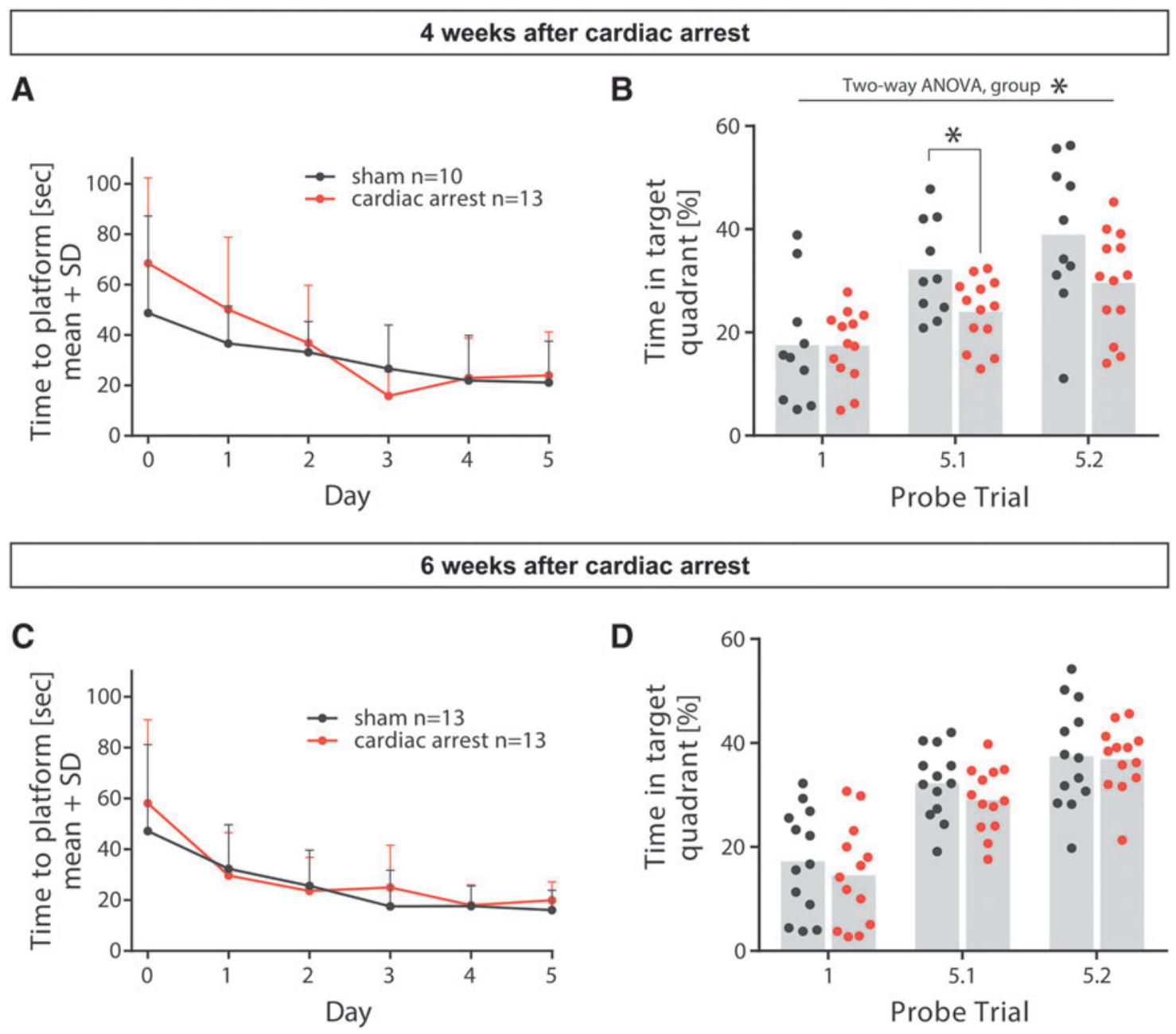

FIG. 2. Neurofunctional assessment 4 and 6 weeks after surgery using Morris water maze. Learning performance of ischemic and control animals was similar at both time points, as indicated by the steady decrease in time to find the platform over the 5 training days $(\mathbf{A}, \mathbf{C})$. Day 0 represents the first training trial on day 1. Four weeks after cardiac arrest and resuscitation, animals showed significant memory impairments compared to controls $(\mathbf{B})$. During both probe trials on day 5 (5.1 and 5.2), they spent less time in the target quadrant, where the platform was located during training, although the difference reached significance only in the first trial. Overall, cardiac arrest animals performed significantly worse than controls, as assessed by a two-way ANOVA. Six weeks after surgery, the difference in memory performance could not be detected anymore (D).

while most grafted cells located in the CA1 segment did not (Fig. 5D, E). None of the grafted cells was found to express glial markers (Supplementary Fig. S5).

\section{Discussion}

\section{Extension of cardiac arrest time to $12 \mathrm{~min}$}

When compared with our previous study [35], extension of no-flow time from 10 to $12 \mathrm{~min}$ resulted in a similar rate of survival, time to ROSC, and administration of adrenaline. However, the extent of histopathological damage was not increased. We performed the quantification 2 days later; thus, it cannot be excluded that we would have found more degenerating cells on day 5. Although, in our protocol, the no-flow time was significantly longer than the usual median of 6-8 min in cardiac arrest models, we reached a higher ROSC and survival rate than the median of $85 \%$ and $60 \%$, respectively [36].

\section{Permanent loss of CA1 neurons following global brain ischemia}

Persistency of neuronal loss in the CA1 segment is in contrast to other studies that showed regeneration of the damaged area following global brain ischemia [37-41]. It was speculated that new neurons originate from progenitor cells residing around the posterior periventricle, which proliferate in response to injury, migrate toward the damaged area, differentiate, and integrate in the existing circuit [37]. In some instances, this repopulation was correlated to a recovery of previously detected learning and memory deficits $[37,39,40]$. However, these studies used a different model of global brain ischemia, induced by vessel occlusion that exclusively affects the brain.

Contrarily, this model of cardiac arrest and resuscitation leads to ischemia and reperfusion injury throughout the body, associated with a systemic inflammatory response 

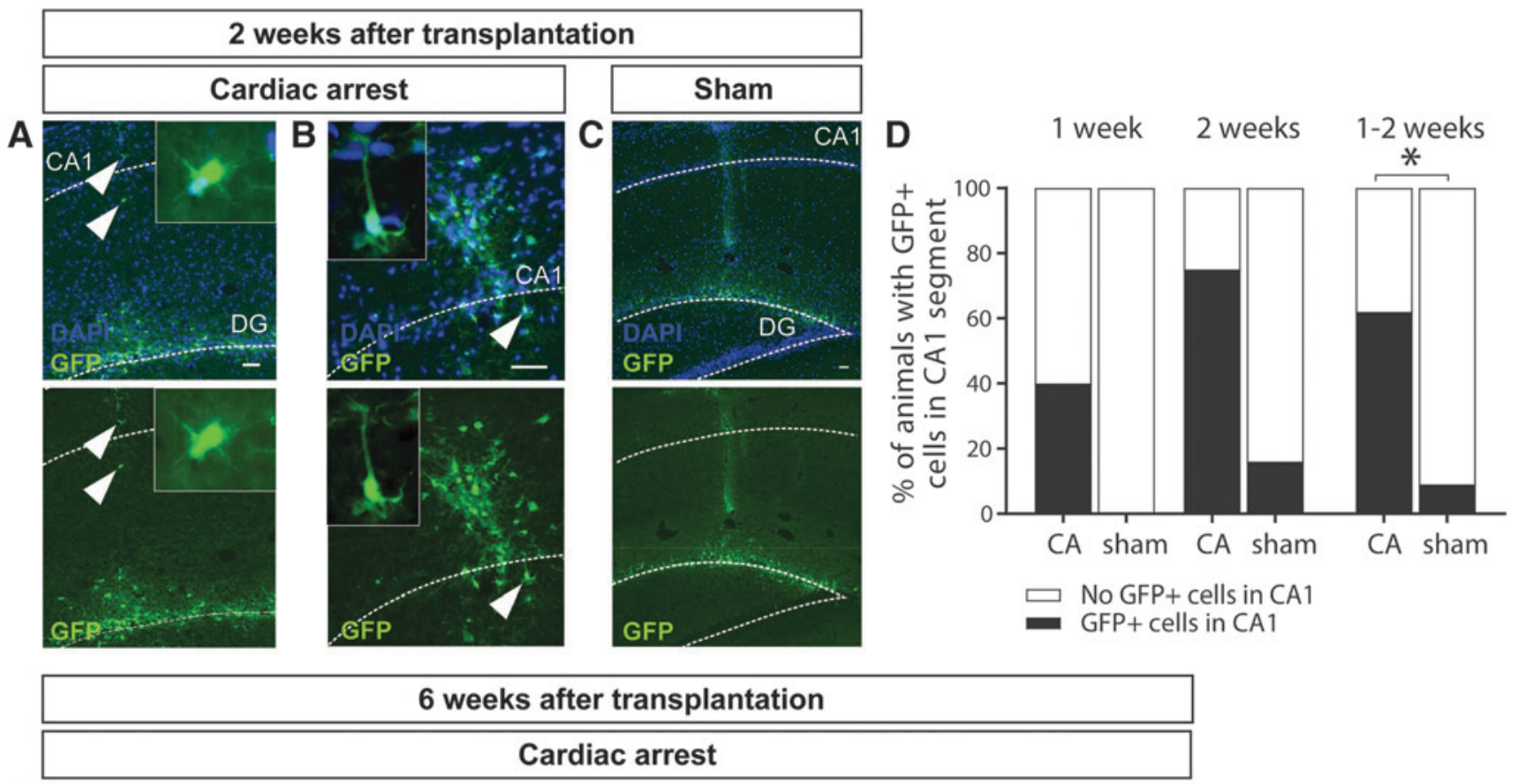

E
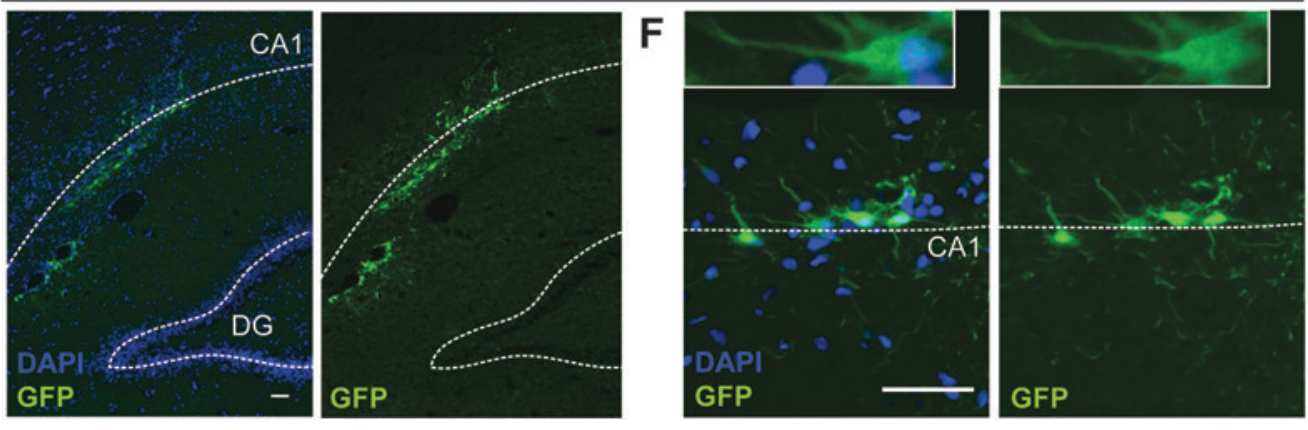

FIG. 3. Location of GFP-expressing cells 2 and 6 weeks after transplantation. Grafted cells were found to accumulate along the granular layer of the DG in both ischemic animals (A) as well as controls (C). However, in case of a damaged CA1 segment, GFP-expressing cells (representative cells identified by arrowheads and depicted in the inserts) showed a stronger tendency to persist in injured area $(\mathbf{A}, \mathbf{B}, \mathbf{E}, \mathbf{F})$. In controls, grafted cells were only found in the CA1 layer during their migration toward the ventrally located dentate gyrus (C). Quantification showed that the probability of finding transplanted cells in the CA1 segment was significantly higher $(* P<0.05)$ in cardiac arrest animals compared to controls (D). Scale bars $50 \mu \mathrm{m}$. GFP, green fluorescent protein.

[14]. These events may lead to a more severe and intricate brain damage, which could negatively impact the endogenous regenerative potential. Since some of the publications are long-term studies of up to 4 months [40], we cannot exclude similar findings if the follow-up time was extended. The majority of vessel occlusion studies first performed behavioral experiments 1-2 weeks after injury [37,39,40,42]. In our experimental model, this is not feasible, since a longer recovery period is needed, as is the case in other cardiac arrest models [43]. Contradictory results generated in specific models of global brain ischemia have been reported before $[44,45]$. Thus, the experimental settings applied to induce global brain ischemia are critically defining the outcome, which makes direct comparisons between the models difficult.

\section{Transient memory deficits after cardiac arrest and resuscitation}

The memory impairments observed at 4 weeks were compensated by 6 weeks after cardiac arrest. Based on the massive hippocampal cell death, we expected more severe impairments in the water maze performance. The transient nature of the observed memory deficits is in accordance to several studies, which correlated it to endogenous regeneration provided by cell replenishment $[37,39,40]$. In this study, this is unlikely, since no repopulation of the damaged area was found.

The striking discrepancy between the histological damage and the moderate cognitive deficits observed could be explained by the involvement of non-hippocampal brain regions in spatial memory performance. These structures might be able to take over the role of the injured area, in accordance to the brain plasticity concept. In addition, the neurofunctional impairments detected with the water maze paradigm might not exclusively be caused by the CA1 cell loss. This would imply that the water maze paradigm involves the activity of hippocampus-unrelated structures. Although both hypotheses are supported by numerous studies in animals and humans [46-51], they are often neglected when focusing on hippocampal dysfunction and its influence on learning and memory. 
A
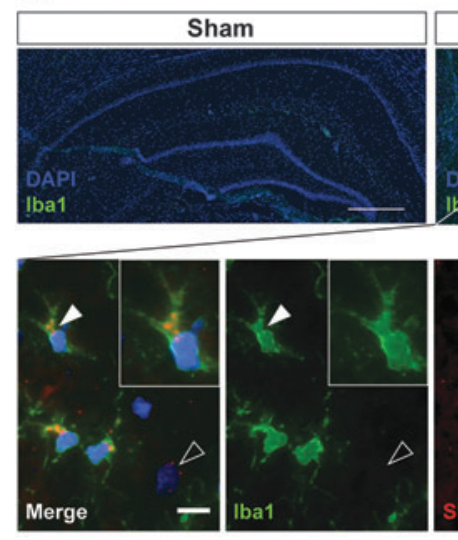

C

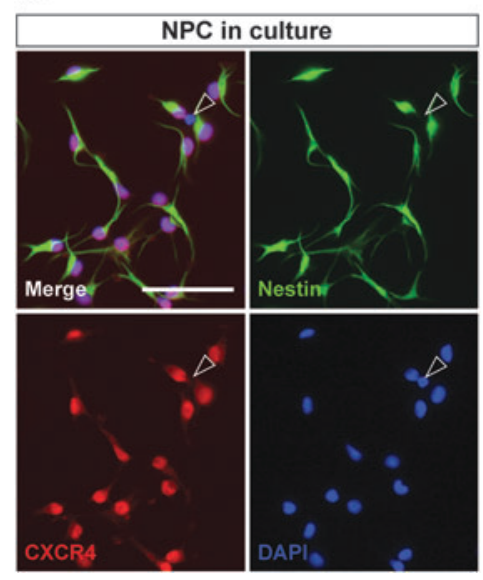

B
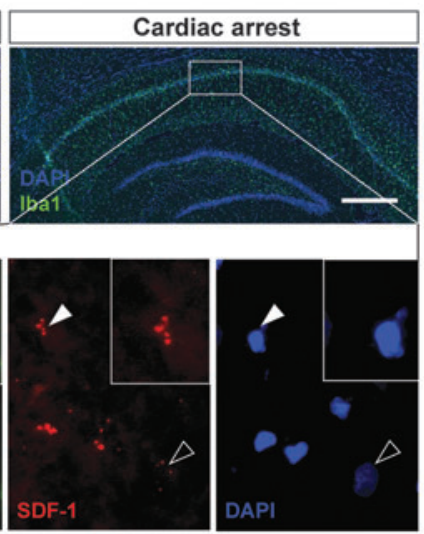
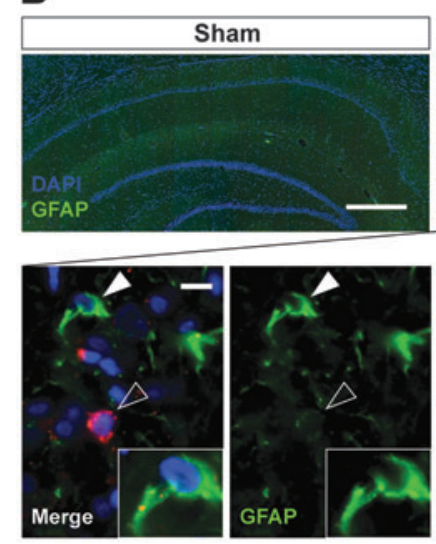
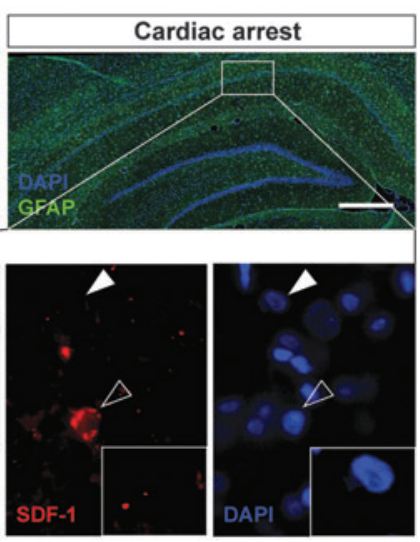

D

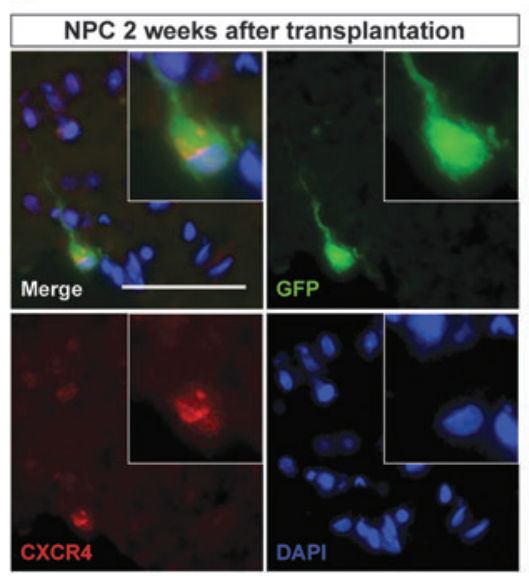

FIG. 4. Expression of the chemotaxis molecule SDF-1 and its receptor CXCR4 by glia cells and transplanted NPCs, respectively. One week after cardiac arrest, Iba1-positive microglia (A) and GFAP-positive astrocytes (B) accumulating in the damaged CA1 segment expressed SDF-1 (white arrowheads), while in sham animals, almost no glia cells were found in the hippocampus. Other SDF-1 expressing cells (blank arrowheads) might be unstained glia cells or neurons, also known to express the chemokine [58]. Cultured nestin-positive NPCs expressed CXCR4 at the time point of transplantation (C), while nestin-negative cells (glia or differentiated neurons) did not (blank arrowheads). Two weeks after transplantation, GFPexpressing NPCs were still positive for CXCR4 (D). Scale bars $500 \mu \mathrm{m}$ (A, B, whole hippocampus), $10 \mu \mathrm{m}$ (A, B, insets) and $50 \mu \mathrm{m}(\mathbf{C}, \mathbf{D})$. CXCR4, CXC chemokine receptor 4; NPC, neural progenitor cell; SDF-1, stromal cell-derived factor 1.

Our difficulties to detect deficits at later time points might also be due to the failure of a too simple water maze protocol to detect differences between groups that get more subtle over time, as has been demonstrated by more challenging paradigms $[42,43,49]$. The protocols applied for behavioral assessment considerably influence the experiment's outcome, and might, in part, explain the differences of our results with other studies describing persisting memory deficits after ischemia [41,42].

We are aware that, for the evaluation of a regenerative treatment, not only histological analyses but also additional long-term functional outcome measures should be considered. However, the effect of cardiac arrest on long-term learning and memory behaviour has still not been investigated thoroughly enough in rodent models, and the few available studies are contradictory, observing both persistent $[41,42]$ and transient $[37,39,40]$ memory deficits. In addition to learning and memory behaviour, sensorimotor analysis after cardiac arrest would be another functional outcome measure. However, in our previous work [52], we did not find any persistent sensorimotor deficit after $8 \mathrm{~min}$ of cardiac arrest, and the same applies to this study. Nevertheless, the described experimental model represents a valuable tool for the evaluation of novel therapeutic strategies, in particular when aiming at an increased neuroregeneration, easily detectable by histological analysis, since no spontaneous repopulation of the damaged CA1 segment was observed.

\section{Retention of transplanted NPCs in the damaged CA1 segment}

We observed viable cells up to 6 weeks after grafting and an increased propensity of the cells to persist in the CA1 segment in ischemic animals. It is known that, following brain damage, endogenous or transplanted neural stem cells (NSCs) migrate to the injury site [53]. This mobilization might be induced by the inflammatory response occurring there. Immune cells, including astrocytes and microglia, produce attractants, which guide the NSCs toward their sources [34,54-56]. The same molecular mechanisms might be responsible for the observed accumulation of transplanted cells in the CA1 segment after cardiac arrest. Indeed, we 
A

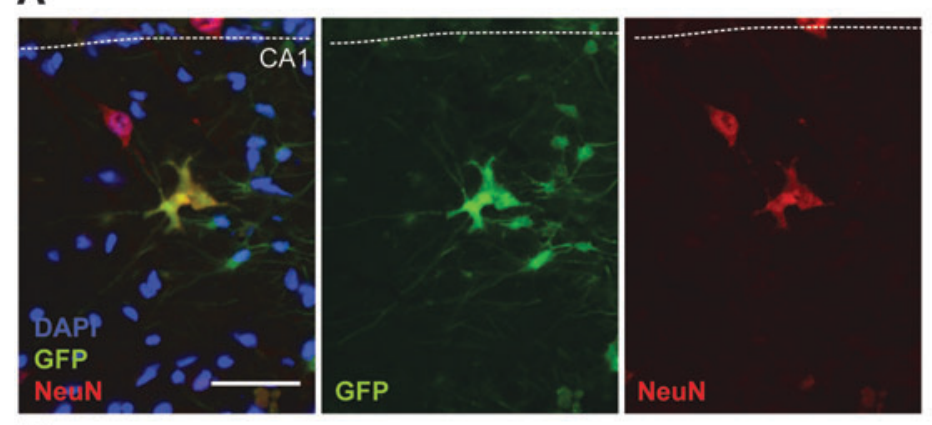

FIG. 5. Phenotypic characterization of GFP-expressing cells 6 weeks after transplantation in cardiac arrest animals. A considerable number of grafted cells were found to express the mature neuron marker NeuN (A), while others were positive for $\beta$ IIItubulin (white arrowheads), specific for immature neurons, (B). Furthermore, some grafted cells remained positive for stem and progenitor cell markers, such as vimentin (white arrowheads) (C) or SOX2 (D), the latter being expressed mainly by cells located in the dentate gyrus (white arrowheads) (D) and less by cells in the CA1 segment (E). Scale bars $50 \mu \mathrm{m}$. SOX2, SRY-box transcription factor 2 .
B
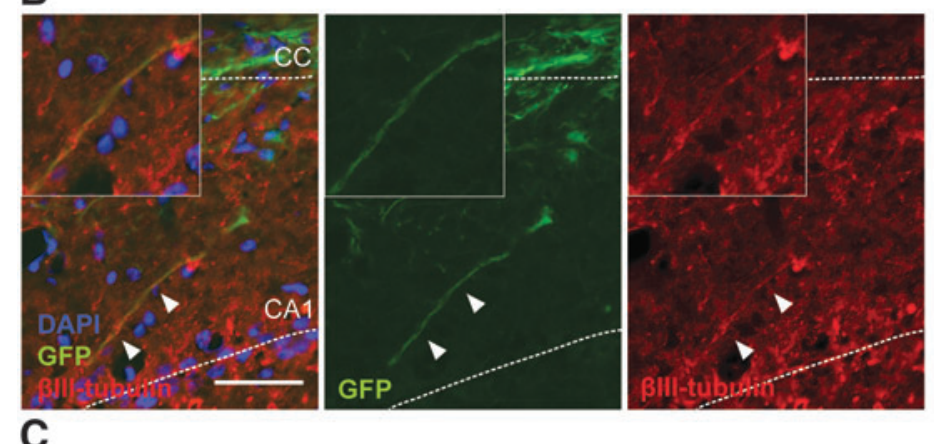

C
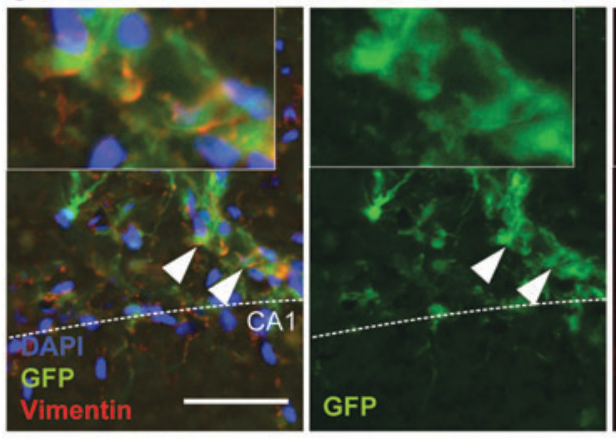

D
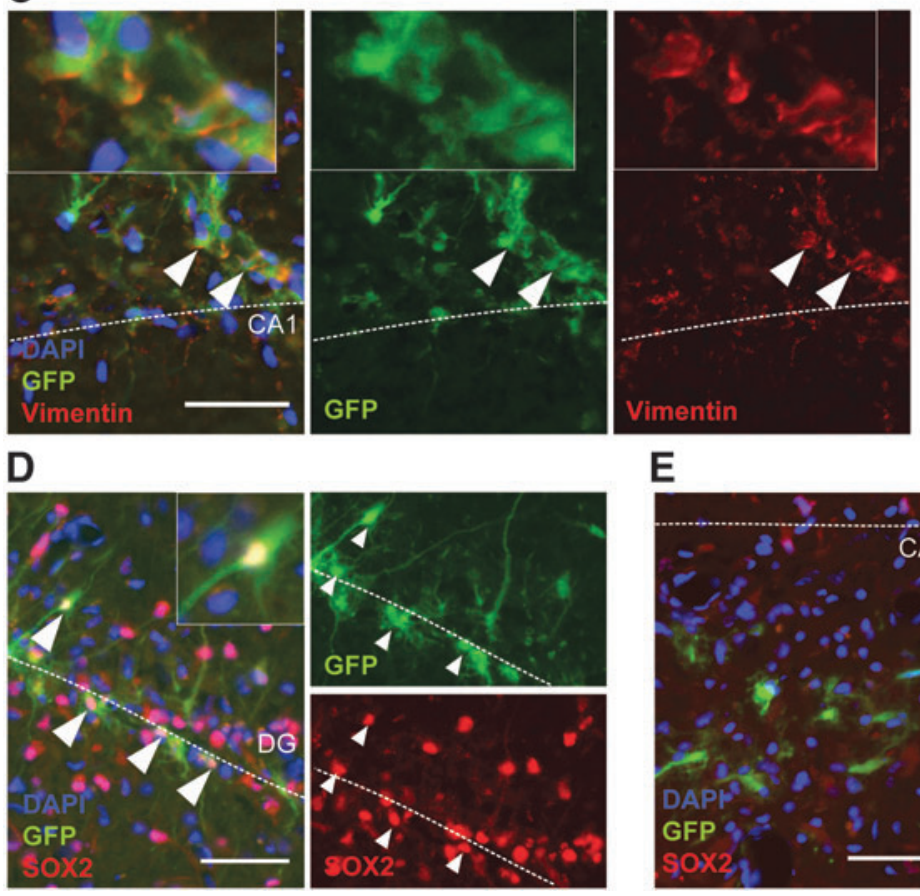

E

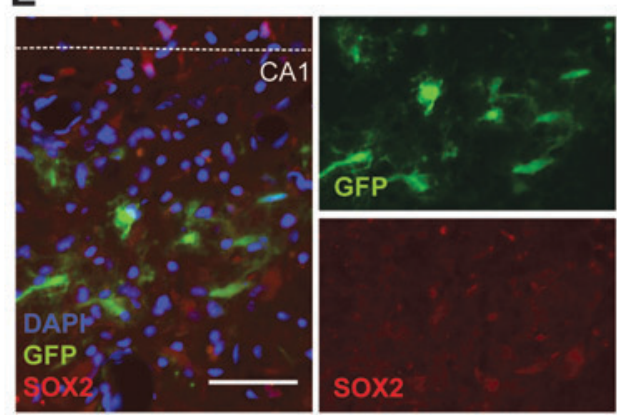

could confirm this hypothesis in our study, by showing the presence of a large number of reactive SDF-1-positive glia in the damaged CA1 segment and the expression of its receptor CXCR4 by the transplanted cells, suggesting a chemotactic-driven retention at the site of injury. However, this mechanism may compete with signals provided by the dentate gyrus, since we documented a large proportion of transplanted cells migrating to its granule cell layer, that is, their endogenous niche.

At the injury site, we documented expression of $\mathrm{NeuN}$ or $\beta$ III-tubulin in a considerable proportion of transplanted cells, confirming their potential to differentiate toward ma- ture neurons. However, an important proportion of NPCs still displayed an undifferentiated phenotype, expressing vimentin and SOX2. These cells have maintained their stem and progenitor identity, but may still influence the regenerative processes through immunomodulation and trophic support [57]. Interestingly, the majority of GFP-expressing cells found in the dentate gyrus expressed SOX2, indicating a stronger stimulus to retain their multipotency, as expected from their stem cell niche environment. Although located in the dentate gyrus and not directly in the damaged area, these cells could potentially have an effect on regeneration through trophic support. 
Overall, the observed long-term survival, the SDF-1driven retention at the damaged area, and the differentiation capacity of transplanted NPCs underline their potential in supporting endogenous regeneration by replacing lost cells.

Limitations of this study include the use of healthy young male animals, which is different from the population of cardiac arrest patients that is mostly composed of older people often affected by comorbidities [12,58]. In addition, we only assessed hippocampal damage, although other brain areas may have been affected by ischemia. Given the lack of differences between sham and cardiac arrest animals at the planned time point we assessed neurofunctional outcome, we were not able to assess the functional effect of grafted NPCs.

\section{Conclusion}

We conclude that NPC transplantation after cardiac arrest is feasible, with documentation of their survival, persistence at the injured site, neuronal differentiation, and therefore potential for cell replacement. Furthermore, we confirmed two of the key consequences of cardiac arrest, loss of CA1 pyramidal neurons and early memory deficits in our experimental model. For studies focusing on cardiac arrest sequelae, our model of global brain ischemia might be more relevant than vessel occlusion models since it recapitulates more accurately the complex cascade of pathological processes occurring in patients.

\section{Acknowledgments}

We acknowledge Prof. Kobayashi (Jichi Medical School, Japan) for providing the GFP-expressing Lewis rat strain. We thank Prof. Hans Rudolf Widmer and Dr. Amiq Gazdhar (University of Bern, Switzerland) for providing several antibodies. Furthermore, we thank Dr. Daniela Casoni and her team from the Experimental Surgery Facility (University of Bern, Switzerland) for providing the necessary facilities to conduct the animal surgeries.

\section{Author Disclosure Statement}

No competing financial interests exist.

\section{Funding Information}

This work was financially supported by the Department of Intensive Care Medicine, Inselspital, University Hospital Bern and by the Swiss National Science Foundation (grant 162583 and 189136).

\section{Supplementary Material}

Supplementary Data

Supplementary Figure S1

Supplementary Figure S2

Supplementary Figure S3

Supplementary Figure S4

Supplementary Figure S5

\section{References}

1. Berdowski J, RA Berg, JG Tijssen and RW Koster. (2010). Global incidences of out-of-hospital cardiac arrest and survival rates: systematic review of 67 prospective studies. Resuscitation 81:1479-1487.

2. Madl C and M Holzer. (2004). Brain function after resuscitation from cardiac arrest. Curr Opin Crit Care 10:213217.

3. Grubb NR, R O'Carroll, SM Cobbe, J Sirel and KAA Fox. (1996). Chronic memory impairments after OHCA. BMJ 313:143-146.

4. Moulaert VR, JA Verbunt, CM van Heugten and DT Wade. (2009). Cognitive impairments in survivors of out-ofhospital cardiac arrest: a systematic review. Resuscitation 80:297-305.

5. Schmidt-Kastner R and TF Freund. (1991). Selective vulnerability of the hippocampus in brain ischemia. Neuroscience 40:599-636.

6. Kirino T. (1982). Delayed neuronal death in the gerbil hippocampus following ischemia. Brain Res 239:57-69.

7. Pulsinelli WA, JB Brierley and F Plum. (1982). Temporal profile of neuronal damage in a model of transient forebrain ischemia. Ann Neurol 11:491-498.

8. Nunn J and H Hodges. (1994). Cognitive deficits induced by global cerebral ischaemia: relationship to brain damage and reversal by transplants. Behav Brain Res 65:1-31.

9. Zola-Morgan S, LR Squire and DG Amaral. (1986). Human amnesia and the medial temporal region: enduring memory impairment following a bilateral lesion limited to field CA1 of the hippocampus. J Neurosci 6:2950-2967.

10. Bernard SA, TW Gray, MD Buist, BM Jones, W Silvester, G Gutteridge and K Smith. (2002). Treatment of comatose survivors of out-of-hospital cardiac arrest with induced hypothermia. N Engl J Med 346:557-563.

11. Hypothermia after Cardiac Arrest Study Group. (2002). Mild therapeutic hypothermia to improve the neurologic outcome after cardiac arrest. N Engl J Med 346:549-556.

12. Nielsen N, J Wetterslev, T Cronberg, D Erlinge, Y Gasche, C Hassager, J Horn, J Hovdenes, J Kjaergaard,et al. (2013). Targeted temperature management at 33 degrees $\mathrm{C}$ versus 36 degrees C after cardiac arrest. N Engl J Med 369:21972206.

13. Donnino MW, LW Andersen, KM Berg, JC Reynolds, JP Nolan, PT Morley, E Lang, MN Cocchi, T Xanthos, CW Callaway and J Soar. (2016). Temperature management after cardiac arrest: an advisory statement by the Advanced Life Support Task Force of the International Liaison Committee on Resuscitation and the American Heart Association Emergency Cardiovascular Care Committee and the Council on Cardiopulmonary, Critical Care, Perioperative and Resuscitation. Circulation 132:2448-2456.

14. Huang L, PM Applegate, JW Gatling, DB Mangus, J Zhang and RL Applegate. (2014). A systematic review of neuroprotective strategies after cardiac arrest: from bench to bedside (part II-comprehensive protection). Med Gas Res 4:10.

15. Geocadin RG, MA Koenig, X Jia, RD Stevens and MA Peberdy. (2008). Management of brain injury after resuscitation from cardiac arrest. Neurol Clin 26:487-506, ix.

16. Nolan JP, RA Berg, S Bernard, BJ Bobrow, CW Callaway, T Cronberg, RW Koster, PJ Kudenchuk, G Nichol,et al. (2017). Intensive care medicine research agenda on cardiac arrest. Intensive Care Med 43:1282-1293.

17. Zhu SZ, V Szeto, MH Bao, HS Sun and ZP Feng. (2018). Pharmacological approaches promoting stem cell-based therapy following ischemic stroke insults. Acta Pharmacol Sin 39:695-712. 
18. Janowski M, DC Wagner and J Boltze. (2015). Stem cellbased tissue replacement after stroke: factual necessity or notorious fiction? Stroke 46:2354-2363.

19. Ding DC, CH Lin, WC Shyu and SZ Lin. (2013). Neural stem cells and stroke. Cell Transplant 22:619-630.

20. Leong KH, LL Zhou, QM Lin, P Wang, L Yao and ZT Huang. (2016). Therapeutic effects of various methods of MSC transplantation on cerebral resuscitation following cardiac arrest in rats. Mol Med Rep 13:3043-3051.

21. Wang T, W Tang, S Sun, T Xu, H Wang, J Guan, Z Huang and MH Weil. (2008). Intravenous infusion of bone marrow mesenchymal stem cells improves brain function after resuscitation from cardiac arrest. Crit Care Med 36:S486S491.

22. Hodges H, P Sowinski, P Fleming, TR Kershaw, JD Sinden, BS Meldrum and JA Gray. (1996). Contrasting effects of fetal CA1 and CA3 hippocampal grafts on deficits in spatial learning and working memory induced by global cerebral ischaemia in rats. Neuroscience 72:959-988.

23. Chu K, M Kim, SW Jeong, SU Kim and BW Yoon. (2003). Human neural stem cells can migrate, differentiate, and integrate after intravenous transplantation in adult rats with transient forebrain ischemia. Neurosci Lett 343:129-133.

24. Chung TN, JH Kim, BY Choi, SP Chung, SW Kwon and SW Suh. (2015). Adipose-derived mesenchymal stem cells reduce neuronal death after transient global cerebral ischemia through prevention of blood-brain barrier disruption and endothelial damage. Stem Cells Transl Med 4:178-185.

25. Sinden JD, F Rashid-Doubell, TR Kershaw, A Nelson, A Chadwick, PS Jat, MD Noble, H Hodges and JA Gray. (1997). Recovery of spatial learning by grafts of a conditionally immortalized hippocampal neuroepithelial cell line into the ischaemia-lesioned hippocampus. Neuroscience 81:599-608.

26. Hodges H, A Nelson, D Virley, TR Kershaw and JD Sinden. (1997). Cognitive deficits induced by global cerebral ischaemia: prospects for transplant therapy. Pharmacol Biochem Behav 56:763-780.

27. Frick T, D Springe, D Grandgirard, SL Leib and $M$ Haenggi. (2016). An improved simple rat model for global cerebral ischaemia by induced cardiac arrest. Neurol Res 38:373-380.

28. Muri L, D Grandgirard, M Buri, M Perny and SL Leib. (2018). Combined effect of non-bacteriolytic antibiotic and inhibition of matrix metalloproteinases prevents brain injury and preserves learning, memory and hearing function in experimental paediatric pneumococcal meningitis. $\mathrm{J}$ Neuroinflammation 15:233.

29. Inoue H, I Ohsawa, T Murakami, A Kimura, Y Hakamata, Y Sato, T Kaneko, M Takahashi, T Okada,et al. (2005). Development of new inbred transgenic strains of rats with LacZ or GFP. Biochem Biophys Res Commun 329:288295.

30. Fath T, YD Ke, P Gunning, J Gotz and LM Ittner. (2009). Primary support cultures of hippocampal and substantia nigra neurons. Nat Protoc 4:78-85.

31. Paxinos G and Watson C. (1988). The Rat Brain in Stereotactic Coordinates. 4th edn. Academic Press, San Diego, CA.

32. Fernández-Arjona MdM, JM Grondona, P Granados-Durán, P Fernández-Llebrez and MD López-Ávalos. (2017). Microglia morphological categorization in a rat model of neuroinflammation by hierarchical cluster and principal components analysis. Front Cell Neurosci 11:235.
33. Lin RC, DF Matesic, M Marvin, RD McKay and O Brustle. (1995). Re-expression of the intermediate filament nestin in reactive astrocytes. Neurobiol Dis 2:79-85.

34. Imitola J, K Raddassi, KI Park, F-J Mueller, M Nieto, YD Teng, D Frenkel, J Li, RL Sidman,et al. (2004). Directed migration of neural stem cells to sites of CNS injury by the stromal cell-derived factor $1 \alpha / \mathrm{CXC}$ chemokine receptor 4 pathway. Proc Natl Acad Sci U S A 101:18117-18122.

35. Glas M, T Frick, D Springe, A Putzu, P Zuercher, D Grandgirard, SL Leib, SM Jakob, J Takala and M Haenggi. (2018). Neuroprotection with the P53-inhibitor pifithrin-mu after cardiac arrest in a rodent model. Shock 49:229-234.

36. Vognsen M, BK Fabian-Jessing, N Secher, B Lofgren, C Dezfulian, LW Andersen and A Granfeldt. (2017). Contemporary animal models of cardiac arrest: a systematic review. Resuscitation 113:115-123.

37. Nakatomi H, T Kuriu, S Okabe, S-i Yamamoto, O Hatano, N Kawahara, A Tamura, T Kirino and M Nakafuku. (2002). Regeneration of hippocampal pyramidal neurons after ischemic brain injury by recruitment of endogenous neural rogenitors. Cell 110:429-441.

38. Schmidt W and KG Reymann. (2002). Proliferating cells differentiate into neurons in the hippocampal CA1 region of gerbils after global cerebral ischemia. Neurosci Lett 334: 153-156.

39. Bendel O, T Bueters, M von Euler, SO Ögren, J Sandin and $\mathrm{G}$ von Euler. (2005). Reappearance of hippocampal CA1 neurons after ischemia is associated with recovery of learning and memory. J Cereb Blood Flow Metab 25:1586-1595.

40. von Euler M, O Bendel, T Bueters, J Sandin and G von Euler. (2006). Profound but transient deficits in learning and memory after global ischemia using a novel water maze test. Behav Brain Res 166:204-210.

41. Langdon KD, S Granter-Button and D Corbett. (2008). Persistent behavioral impairments and neuroinflammation following global ischemia in the rat. Eur J Neurosci 28: 2310-2318.

42. Hartman RE, JM Lee, GJ Zipfel and DF Wozniak. (2005). Characterizing learning deficits and hippocampal neuron loss following transient global cerebral ischemia in rats. Brain Res 1043:48-56.

43. Weihs W, AM Warenits, F Ettl, IA Magnet, U Teubenbacher, A Hilpold, A Schober, C Testori, A Tiboldi, et al. (2016). Reduced long-term memory in a rat model of 8 minutes ventricular fibrillation cardiac arrest: a pilot trial. BMC Vet Res 12:103.

44. Matchett GA, JB Calinisan, GC Matchett, RD Martin and JH Zhang. (2007). The effect of granulocyte-colony stimulating factor in global cerebral ischemia in rats. Brain Res 1136:200-207.

45. Popp E, T Rabsahl, A Schneider, N Russ, F Spohr, P Vogel, BW Bottiger and P Teschendorf. (2009). Intracerebroventricular application of granulocyte colony-stimulating factor after cardiac arrest does not promote beneficial effects on cerebral recovery after cardiac arrest in rats. Resuscitation 80:478-483.

46. Eichenbaum H. (2000). A cortical-hippocampal system for declarative memory. Nat Rev Neurosci 1:41-50.

47. Pouzet B, WN Zhang, J Feldon and JN Rawlins. (2002). Hippocampal lesioned rats are able to learn a spatial position using non-spatial strategies. Behav Brain Res 133:279-291.

48. Gerlai RT, A McNamara, S Williams and HS Phillips. (2002). Hippocampal dysfunction and behavioral deficit in the water maze in mice: an unresolved issue? Brain Res Bull 57:3-9. 
49. Mehla J, SH Deibel, J Faraji, T Saito, TC Saido, MH Mohajerani and RJ McDonald. (2019). Looking beyond the standard version of the Morris water task in the assessment of mouse models of cognitive deficits. Hippocampus 29: 3-14.

50. Tulving E and HJ Markowitsch. (1997). Memory beyond the hippocampus. Curr Opin Neurobiol 7:209-216.

51. Grubb NR, KA Fox, K Smith, J Best, A Blane, KP Ebmeier, MF Glabus and RE O'Carroll. (2000). Memory impairment in out-of-hospital cardiac arrest survivors is associated with global reduction in brain volume, not focal hippocampal injury. Stroke 31:1509-1514.

52. Zuercher P, D Springe, D Grandgirard, SL Leib, M Grossholz, S Jakob, J Takala and M Haenggi. (2016). A randomized trial of the effects of the noble gases helium and argon on neuroprotection in a rodent cardiac arrest model. BMC Neurol 16:43.

53. Garzon-Muvdi T and A Quinones-Hinojosa. (2009). Neural stem cell niches and homing: recruitment and integration into functional tissues. ILAR J 51:3-23.

54. Gonzalez R, MH Hamblin and JP Lee. (2016). Neural stem cell transplantation and CNS diseases. CNS Neurol Disord Drug Targets 15:881-886.

55. Merino JJ, V Bellver-Landete, MJ Oset-Gasque and B Cubelos. (2015). CXCR4/CXCR7 molecular involvement in neuronal and neural progenitor migration: focus in CNS repair. J Cell Physiol 230:27-42.

56. Belmadani A, PB Tran, D Ren and RJ Miller. (2006). Chemokines regulate the migration of neural progenitors to sites of neuroinflammation. J Neurosci 26:3182-3191.

57. Martino G and S Pluchino. (2006). The therapeutic potential of neural stem cells. Nat Rev Neurosci 7:395-406.

58. Hirlekar G, M Jonsson, T Karlsson, J Hollenberg, P Albertsson and J Herlitz. (2018). Comorbidity and survival in out-of-hospital cardiac arrest. Resuscitation 133:118-123.

Address correspondence to: Stephen L. Leib, MD Neuroinfection Laboratory Institute for Infectious Diseases University of Bern Friedbühlstrasse 51

Bern 3001 Switzerland

E-mail: stephen.leib@ifik.unibe.ch

Received for publication August 27, 2019

Accepted after revision January 21, 2020

Prepublished on Liebert Instant Online January 22, 2020 